\title{
Three-Directional Box-Splines: Characterization and Efficient Evaluation
}

\author{
Laurent Condat, Student Member, IEEE, and Dimitri Van De Ville, Member, IEEE
}

\begin{abstract}
We propose a new characterization of three-directional box-splines, which are well adapted for interpolation and approximation on hexagonal lattices. Inspired by a construction already applied with success for exponential splines [1] and hex-splines [2], we characterize a box-spline as a convolution of a generating function, which is a Green function of the spline's associated differential operator, and a discrete filter that plays the role of a localization operator. This process leads to an elegant analytical expression of three-directional box-splines. It also brings along a particularly efficient implementation.
\end{abstract}

Index Terms-Approximation, box-splines, hexagonal sampling, interpolation, three-directional mesh.

\section{INTRODUCTION}

$\mathbf{T}$ HE representation of a digital signal by means of a discrete/continuous model is essential for common tasks such as interpolation and resampling. For images and other two-dimensional (2-D) data, polynomial spline models based on B-splines are particularly popular, mainly due to their simplicity and excellent approximation capabilities [3].

For image data sampled on the traditional Cartesian lattice, separable B-splines can be obtained in a straightforward way using tensor products of one-dimensional (1-D) B-splines. However, in the case of sampling on a hexagonal lattice (aka three-directional mesh), separable B-splines are incapable of exploiting the highly praised isotropy and twelve-fold symmetry of this sampling scheme [4], [5]. Box-splines are a multidimensional extension of 1-D splines [6] that have found practical applications in geometric modeling, multiscale representation, and many other fields. Among the large box-spline family, three-directional (nonseparable) box-splines are particularly suitable for hexagonal lattices. They have been successfully applied in numerous problems where hexagonally sampled data are handled [7].

Early algorithms to evaluate box-spline surfaces were very memory consuming and only resulted into an approximation of the surface within a given tolerance [8], [9]. Later, more efficient methods were proposed based on the recursive properties of box-splines [10]-[12]. Here, we propose a new characterization of three-directional box-splines that provides us with a

Manuscript received November 25, 2005; revised January 11, 2006. This work was supported in part by Région Rhône-Alpes under the EURODOC grant. The associate editor coordinating the review of this manuscript and approving it for publication was Prof. Philippe Salembier.

L. Condat is with the Laboratory of Images and Signals (LIS), Institut National Polytechnique de Grenoble (INPG), 38031 Grenoble, France (e-mail: laurent.condat@lis.inpg.fr).

D. Van De Ville is with the Biomedical Imaging Group (BIG), École Polytechnique Fédérale de Lausanne (EPFL), CH-1015 Lausanne, Switzerland (e-mail: dimitri.vandeville@epfl.ch).

Digital Object Identifier 10.1109/LSP.2006.871852

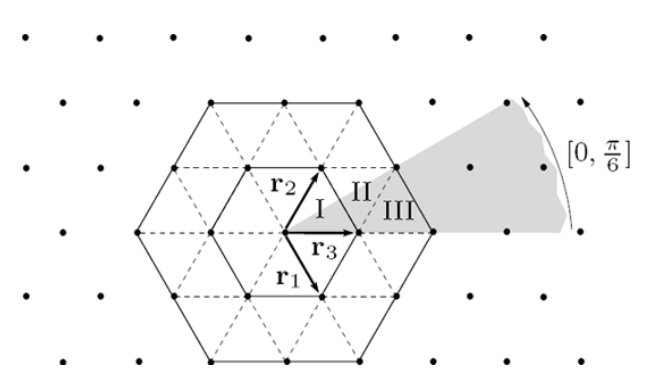

Fig. 1. Hexagonal lattice is generated using integer combinations of the vectors $\mathbf{r}_{1}, \mathbf{r}_{2}$, and $\mathbf{r}_{3}$. The hexagonal support of the first two box-splines $\chi^{1}(\mathbf{x})$ and $\chi^{2}(\mathbf{x})$ has been indicated. Box-splines are polynomial inside each triangle. Using the twelve-fold symmetry, $\chi^{1}$ and $\chi^{2}$ have only to be known in the triangles I, II, and III that intersect the sector $[0, \pi / 6]$.

closed analytical formula, as well as an efficient implementation scheme. To this aim, we derive an explicit form of the generating function, which is the Green function of a three-directional differential operator associated with box-splines. Then, the box-spline can be expressed as the convolution of the generating function with a discrete filter, which plays the role of a localization operator. A similar construction was already applied on the Cartesian lattice to generalized polynomial splines (i.e., exponential splines and L-splines [1]) and to the design of hex-splines, another family of hexagonal splines [2].

\section{BoX-SPlinES ON THE HeXagonal Lattice}

\section{A. Mathematical Preliminaries}

A 2-D lattice is a set of points of the plane, characterized by two linearly independent vectors $\mathbf{v}_{1}$ and $\mathbf{v}_{2}$, grouped in a matrix $\mathbf{R}=\left[\mathbf{v}_{1} \mathbf{v}_{2}\right]$, such that the lattice sites are the locations $\mathbf{R k}$ for every $\mathbf{k} \in \mathbb{Z}^{2 \times 1}$. Within this letter, we define the vectors $\mathbf{e}_{1}=\left[\begin{array}{ll}1 & 0\end{array}\right]^{\mathrm{T}}, \mathbf{e}_{2}=\left[\begin{array}{ll}0 & 1\end{array}\right]^{\mathrm{T}}$, and those shown in Fig. 1 as

$$
\mathbf{r}_{1}=\left[\begin{array}{c}
\frac{1}{2} \\
-\frac{\sqrt{3}}{2}
\end{array}\right], \quad \mathbf{r}_{2}=\left[\begin{array}{c}
\frac{1}{2} \\
\frac{\sqrt{3}}{2}
\end{array}\right], \quad \mathbf{r}_{3}=\left[\begin{array}{l}
1 \\
0
\end{array}\right] .
$$

The Cartesian lattice is then obtained for $\mathbf{R}=\left[\mathbf{e}_{1} \mathbf{e}_{2}\right]$ and the regular hexagonal lattice, as in Fig. 1, for $\mathbf{R}=\left[\begin{array}{rr}\mathbf{r}_{1} & \mathbf{r}_{2}\end{array}\right]$.

Bivariate functions are equivalently denoted as $f\left(x_{1}, x_{2}\right)$, $x_{1}, x_{2} \in \mathbb{R}$, or $f(\mathbf{x})$, where $\mathbf{x}=\left[\begin{array}{ll}x_{1} & x_{2}\end{array}\right]^{\mathrm{T}}$ is interpreted as a vector in $\mathbb{R}^{2}$. The Fourier transform of a function $f(\mathbf{x}) \in$ $L_{2}\left(\mathbb{R}^{2}\right)$ is defined as $\hat{f}(\boldsymbol{\omega})=\int_{\mathbb{R}^{2}} f(\mathbf{x}) \exp (-j\langle\boldsymbol{\omega}, \mathbf{x}\rangle) d \mathbf{x}$, where $\langle\boldsymbol{\omega}, \mathbf{x}\rangle=\boldsymbol{\omega}^{\mathrm{T}} \mathbf{x}$ is the usual inner product of vectors.

A 2-D discrete signal is denoted as $s[\mathbf{k}]=s\left[k_{1}, k_{2}\right]$, $k_{1}, k_{2} \in \mathbb{Z}$. Its representation in the continuous domain, associated with the lattice sites $\mathbf{R k}$, is a weighted Dirac comb: $s(\mathbf{x})=\sum_{\mathbf{k} \in \mathbb{Z}^{2}} s[\mathbf{k}] \delta(\mathbf{x}-\mathbf{R k})$. Consequently, its Fourier transform is defined accordingly as $\hat{s}(\boldsymbol{\omega})=\sum_{\mathbf{k} \in \mathbb{Z}^{2}} s[\mathbf{k}] \exp (-j\langle\boldsymbol{\omega}, \mathbf{R k}\rangle)$. For $\mathbf{z}=\exp \left(-j \mathbf{R}^{\mathrm{T}} \boldsymbol{\omega}\right)$, 

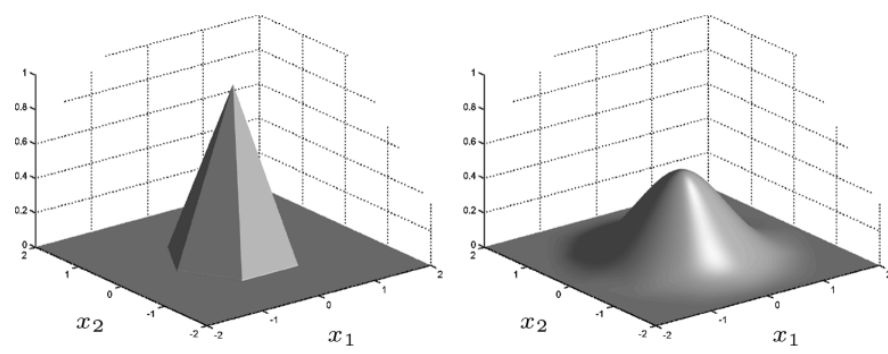

Fig. 2. First two box-splines (left) $\chi^{1}(\mathbf{x})$ and (right) $\chi^{2}(\mathbf{x})$.

we get the $\mathcal{Z}$-transform of $s$ as $S(\mathbf{z})=\sum_{\mathbf{k} \in \mathbb{Z}^{2}} s[\mathbf{k}] \mathbf{z}^{-\mathbf{k}}\left(\mathbf{z}^{-\mathbf{k}}\right.$ means $\left.z_{1}^{-k_{1}} z_{2}^{-k_{2}}\right)$. Convolutions are denoted by $*$.

\section{B. Definition}

A 2-D box-spline model defined on a lattice $\mathbf{R}$ has the form

$$
f(\mathbf{x})=\sum_{\mathbf{k} \in \mathbb{Z}^{2}} c[\mathbf{k}] \varphi_{\Xi}(\mathbf{x}-\mathbf{R} \mathbf{k}), \quad \mathbf{x} \in \mathbb{R}^{2}
$$

where $c[\mathbf{k}]$ are the box-spline coefficients that are weights for the box-spline basis functions $\varphi_{\Xi}(\mathbf{x})$, placed on every lattice site. They can be computed to ensure a desired property, typically that $f$ interpolates a discrete available signal $s$ (i.e., $f(\mathbf{R k})=$ $s[\mathbf{k}]$ for every $\mathbf{k})$. The box-spline $\varphi_{\Xi}(\mathbf{x})$ depends on a concatenated matrix of $N$ vectors $\Xi=\left[\mathbf{v}_{1} \cdots \mathbf{v}_{N}\right](N \geq 2)$ and can be defined as follows [6]. If $\Xi=\left[\begin{array}{ll}\mathbf{v}_{1} & \mathbf{v}_{2}\end{array}\right]$, then

$$
\varphi_{\left[\mathbf{v}_{1} \mathbf{v}_{2}\right]}(\mathbf{x})= \begin{cases}\frac{1}{|\operatorname{det}(\Xi)|}, & \text { if } \Xi^{-1} \mathbf{x} \in[0,1)^{2} \\ 0, & \text { otherwise }\end{cases}
$$

and inductively, $\varphi_{\Xi \cup[\mathbf{v}]}(\mathbf{x})=\int_{0}^{1} \varphi_{\Xi}(\mathbf{x}-t \mathbf{v}) d t$.

Therefore, we have the normalization $\int_{\mathbb{R}^{2}} \varphi \Xi=1$ and the convolution property $\varphi \Xi_{1} \cup \Xi_{2}=\varphi \Xi_{1} * \varphi \Xi_{2}$.

On a hexagonal lattice, box-splines can be constructed using the three vectors $\mathbf{r}_{1}, \mathbf{r}_{2}$, and $-\mathbf{r}_{3}$. In particular, we define the so-called Courant element [6] as $\chi^{1}=(\sqrt{3} / 2) \varphi_{\left[\mathbf{r}_{1} \mathbf{r}_{2}-\mathbf{r}_{3}\right]}$, where we have changed the normalization toward the density of the lattice, i.e., $|\operatorname{det} \mathbf{R}|=\sqrt{3} / 2$. Further on, higher orders are obtained as $\chi^{n}=(2 / \sqrt{3}) \chi^{n-1} * \chi^{1}, n>1$. Their expression in the Fourier domain is

$$
\begin{aligned}
\hat{\chi}^{n}(\boldsymbol{\omega}) & =\frac{\sqrt{3}}{2}\left(\frac{\exp \left(j\left\langle\boldsymbol{\omega}, \mathbf{r}_{3}\right\rangle\right) \prod_{i=1}^{3} 1-\exp \left(-j\left\langle\boldsymbol{\omega}, \mathbf{r}_{i}\right\rangle\right)}{\left(j\left\langle\boldsymbol{\omega}, \mathbf{r}_{1}\right\rangle\right)\left(j\left\langle\boldsymbol{\omega}, \mathbf{r}_{2}\right\rangle\right)\left(j\left\langle\boldsymbol{\omega}, \mathbf{r}_{3}\right\rangle\right)}\right)^{n} \\
& =\frac{\sqrt{3}}{2} \prod_{i=1}^{3} \operatorname{sinc}\left(\frac{\left\langle\boldsymbol{\omega}, \mathbf{r}_{i}\right\rangle}{2}\right)^{n}
\end{aligned}
$$

where $\operatorname{sinc}(x)=\sin (x) / x$. The box-splines $\chi^{n}(\mathbf{x})$ have several attractive properties, such as a hexagonal compact support and twelve-fold symmetry, as illustrated in Figs. 1 and 2. In the next section, we provide closed analytical formulas for these boxsplines in the spatial domain.

\section{DIFFERENTIAL CHARACTERIZATION OF BOX-SPLINES}

\section{A. B-Spline Refresher}

In the 1-D case, a polynomial spline $f(x)$ for uniformly sampled data can be expressed similarly to (2) as $f(x)=\sum_{k \in \mathbb{Z}} c[k] \beta^{n}(x-k) . \beta^{n}$ is the causal B-spline of degree $n \in \mathbb{N}$, which can be defined in the spatial domain as

$$
\beta^{n}(x)=\Delta^{n+1} * \frac{(x)_{+}^{n}}{n !} .
$$

We identify $\Delta^{n}$ as the $n$th iterate of the finite difference filter, which is usually expressed in the $\mathcal{Z}$-domain as $\Delta^{n}(z)=\left(1-z^{-1}\right)^{n}$. Further on, we have the one-sided power function $(x)_{+}^{n}=\left\{x^{n}\right.$, for $x>0 ; 0$, otherwise $\}$. The filtering process acts as a localization operator on the power function, i.e., $\beta^{n}$ has a finite support. The term $(x)_{+}^{n} / n$ ! is also called the generating function, and it corresponds to the (causal) Green function of the differential operator $\mathrm{L}^{n}=\mathrm{d}^{n} / \mathrm{d} x^{n}$, i.e., the function $\rho(x)$ such that $\mathrm{L}^{n}\{\rho\}(x)=\delta(x)$. This means that a polynomial spline of degree $n$, when differentiated $n+1$ times, is a weighted Dirac comb.

On the 2-D Cartesian lattice, we can easily use tensor-product B-splines: $\beta^{n}(\mathbf{x})=\beta^{n}\left(x_{1}\right) \beta^{n}\left(x_{2}\right)$. Then, the associated differential operator is

$$
\mathrm{L}^{n}=\frac{\partial^{2 n}}{\partial x_{1}^{n} \partial x_{2}^{n}}=\mathrm{D}_{\mathbf{e}_{1}}^{n} \mathrm{D}_{\mathbf{e}_{2}}^{n} \stackrel{\mathcal{F}}{\longleftrightarrow}\left(j\left\langle\boldsymbol{\omega}, \mathbf{e}_{1}\right\rangle\right)^{n}\left(j\left\langle\boldsymbol{\omega}, \mathbf{e}_{2}\right\rangle\right)^{n}
$$

where $\mathrm{D}_{\mathbf{v}} f(\mathbf{x})=\lim _{t \rightarrow 0}(f(\mathbf{x}+t \mathbf{v})-f(\mathbf{x})) / t$. In that case, the (separable) generating function is $(\mathbf{x})_{+}^{n} /(n !)^{2}=$ $\left(x_{1}\right)_{+}^{n}\left(x_{2}\right)_{+}^{n} /(n !)^{2}$, and the corresponding localization operator $\Delta^{n}(\mathbf{z})=\Delta^{n}\left(z_{1}\right) \Delta^{n}\left(z_{2}\right)$.

\section{B. From Differential Operators to Generating Functions}

Inspired by the B-spline construction using Green functions, we propose an extension for the box-splines on the hexagonal lattice. For this purpose, we introduce the three-directional differential operator $\mathrm{L}^{n}=(2 / \sqrt{3}) \mathrm{D}_{\mathbf{r}_{1}}^{n} \mathrm{D}_{\mathbf{r}_{2}}^{n} \mathrm{D}_{\mathbf{r}_{3}}^{n}, n \geq 1$. Its Fourier transform, in the sense of the distributions, is

$$
\widehat{\mathrm{L}}^{n}(\boldsymbol{\omega})=\frac{2}{\sqrt{3}}\left(j\left\langle\boldsymbol{\omega}, \mathbf{r}_{1}\right\rangle\right)^{n}\left(j\left\langle\boldsymbol{\omega}, \mathbf{r}_{2}\right\rangle\right)^{n}\left(j\left\langle\boldsymbol{\omega}, \mathbf{r}_{3}\right\rangle\right)^{n} \text {. }
$$

Proposition: A Green function $\rho^{n}(\mathbf{x})$ of the operator $\mathrm{L}^{n}, n \geq$ 1 is given by

$$
\rho^{n}(\mathbf{x})=\sum_{i=0}^{n-1}\left(\begin{array}{c}
n-1+i \\
i
\end{array}\right) \mu^{n-1-i, 2 n-1+i}(\mathbf{x})
$$

where

$$
\mu^{n_{1}, n_{2}}\left(x_{1}, x_{2}\right)=\frac{1}{n_{1} ! n_{2} !}\left(\frac{2\left|x_{2}\right|}{\sqrt{3}}\right)^{n_{1}}\left(x_{1}-\frac{\left|x_{2}\right|}{\sqrt{3}}\right)_{+}^{n_{2}} .
$$

The proof is given in the Appendix. Notice that the functions $\mu^{n_{1}, n_{2}}$ and $\rho^{n}$ all have the same wedge-like support; they are causal in $x_{1}$ and symmetric in $x_{2}$, as illustrated in Fig. 3.

\section{From Generating Functions to Box-Splines}

In the Fourier domain, the generating function $\rho^{n}$ corresponds to $\hat{\chi}^{n}$ without its numerator in (4). The remaining term can be identified by introducing the discrete filter

$$
\Delta(\mathbf{z})=\left(1-z_{1}^{-1}\right)\left(1-z_{2}^{-1}\right)\left(z_{1} z_{2}-1\right)
$$




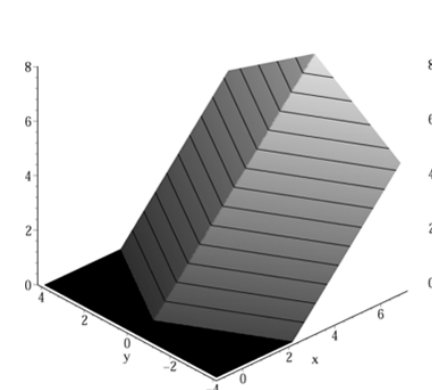

(a)

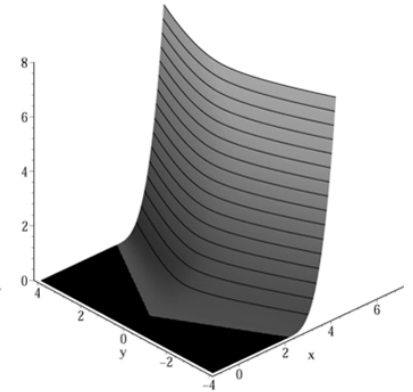

(b)
Fig. 3. Green functions (a) $\rho^{1}=\mu^{0,1}$ and (b) $\rho^{2}=\mu^{1,3}+2 \mu^{0,4}$, which serve to generate the box-splines $\chi^{1}$ and $\chi^{2}$.

Using the property $\mathbf{r}_{3}=\mathbf{r}_{1}+\mathbf{r}_{2}$, we find that $\widehat{\Delta}^{n}(\boldsymbol{\omega})=$ $\Delta\left(\exp \left(j\left\langle\boldsymbol{\omega}, \mathbf{r}_{1}\right\rangle\right), \exp \left(j\left\langle\boldsymbol{\omega}, \mathbf{r}_{2}\right\rangle\right)\right)^{n}$ is exactly the numerator of (4). We can explicitly find the filter coefficients of $\Delta^{n}$ by expanding the $n$th power of the $\mathcal{Z}$-transform of (11). By collecting the coefficient in front of the term $z_{1}^{-k_{1}} z_{2}^{-k_{2}}$, we get for every $k_{1}, k_{2} \in \mathbb{Z}$

$$
\begin{aligned}
& \Delta^{n}\left[k_{1}, k_{2}\right]=\sum_{i=\max \left(k_{1}, k_{2}, 0\right)}^{\min \left(n+k_{1}, n+k_{2}, n\right)}(-1)^{k_{1}+k_{2}+i} \\
& \times\left(\begin{array}{c}
n \\
i-k_{1}
\end{array}\right)\left(\begin{array}{c}
n \\
i-k_{2}
\end{array}\right)\left(\begin{array}{c}
n \\
i
\end{array}\right) .
\end{aligned}
$$

By arranging the $\Delta^{n}[\mathbf{k}]$ at the lattice sites $\mathbf{R k}=k_{1} \mathbf{r}_{1}+k_{2} \mathbf{r}_{2}$, we can represent the first two localization filters as

$$
\Delta=\begin{array}{cccccc}
1 & \begin{array}{c}
1 \\
-1
\end{array} 0^{-1} & -2 & 1 \\
-1 & 1 & \Delta^{2}= & 2 & 2 & -2 \\
2 & -6 & 2 & 1 \\
-2 & 2 & 2 & -2 \\
1 & -2 & 1
\end{array}
$$

Putting together (11) and (8) with the fact that $\widehat{\mathrm{L}}^{n}(\boldsymbol{\omega}) \widehat{\rho}^{n}(\boldsymbol{\omega})=$ 1 , we find that $\widehat{\chi}^{n}(\boldsymbol{\omega})=\widehat{\Delta}^{n}(\boldsymbol{\omega}) \widehat{\rho}^{n}(\boldsymbol{\omega})$. Therefore, we obtain the characterization

$$
\chi^{n}(\mathbf{x})=\Delta^{n} * \rho^{n}(\mathbf{x})=\sum_{\mathbf{k} \in \mathbf{Z}^{2}} \Delta^{n}[\mathbf{k}] \rho^{n}(\mathbf{x}-\mathbf{R} \mathbf{k}) .
$$

The complete analytical expression of $\chi^{n}(\mathbf{x}), n \geq 1$ can then be written as $\chi^{n}\left(x_{1}, x_{2}\right)=$

$$
\begin{aligned}
\sum_{k_{1}, k_{2}=-n}^{n} \sum_{i=\max \left(k_{1}, k_{2}, 0\right)}^{\min \left(n+k_{1}, n+k_{2}, n\right)}(-1)^{k_{1}+k_{2}+i}\left(\begin{array}{c}
n \\
i-k_{1}
\end{array}\right)\left(\begin{array}{c}
n \\
i-k_{2}
\end{array}\right)\left(\begin{array}{c}
n \\
i
\end{array}\right) \\
\quad \times \sum_{d=0}^{n-1}\left(\begin{array}{c}
n-1+d \\
d
\end{array}\right) \frac{1}{(2 n-1+d) !(n-1-d) !} \\
\quad \times \mid \frac{2 x_{2}}{\sqrt{3}}+k_{1}-k_{2}
\end{aligned} \mid \begin{aligned}
& n-1-d \\
& \quad \times\left(x_{1}-\frac{k_{1}+k_{2}}{2}-\left|\frac{x_{2}}{\sqrt{3}}+\frac{k_{1}-k_{2}}{2}\right|\right)_{+}^{2 n-1+d}
\end{aligned}
$$

\section{IMPLEMENTATION ISSUES}

\section{A. Generic Case}

Equation (15) provides us with an efficient way to evaluate at any point $\mathbf{x}$, any three-directional box-spline $\chi^{n}$. Notice that the power functions grow rapidly, as shown in Fig. 3, which could lead to problems of numerical stability. A simple remedy consists of evaluating $\chi^{n}$ only for $x_{1} \leq 0$, which exploits the causality of $\rho^{n}$ in $x_{1}$ and the symmetry $\chi^{n}\left(x_{1}, x_{2}\right)=\chi^{n}\left(-x_{1}, x_{2}\right)$. The following Matlab code performs box-spline evaluations for a list of points $(\mathrm{x}[\mathrm{m}], \mathrm{y}[\mathrm{m}])$, indexed by $\mathrm{m}$. The twelve-fold symmetry is used to fold coordinates into the sector $[5 \pi / 6, \pi]$, where the number of evaluations of the power functions is minimal. We use the coordinates $(u, v)$ in the basis $\left(\mathbf{r}_{1}, \mathbf{r}_{2}\right)$, instead of the coordinates $(x, y)$ in the canonical basis $\left(\mathbf{e}_{1}, \mathbf{e}_{2}\right)$. nchoosek $(\mathrm{n}, \mathrm{k})$ gives the binomial coefficient $(n, k)$.

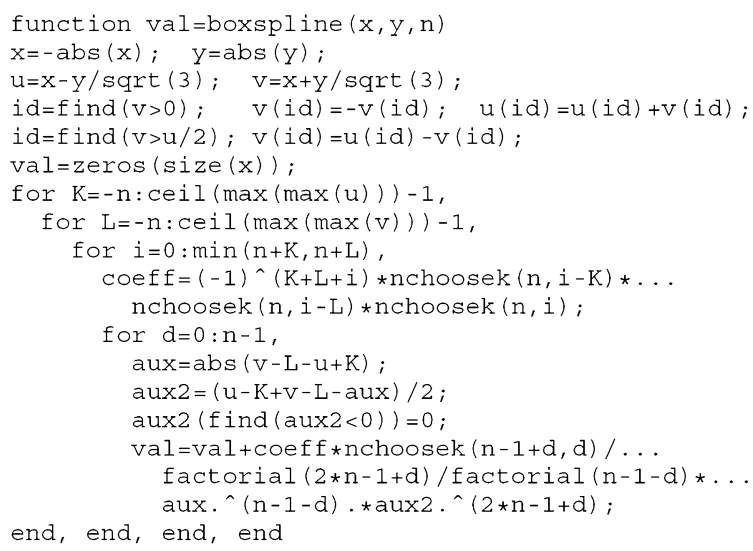

This code was used to generate the plots in Fig. 2. The computational complexity is polynomial in $n$, compared to exponential for recursive methods in the literature [10]-[12]. For example, the evaluation boxspline $(1,1,3)$ took $0.002 \mathrm{~s}$, while $47 \mathrm{~s}$ were required for the same operation using the Matlab code proposed in [11] (that can evaluate any box-spline, not just the three-directional ones).

\section{B. Further Optimization for Fixed $n$}

For evaluating a box-spline $\chi^{n}$ of fixed $n$, an attractive hybrid analytical/numerical implementation consists in determining the polynomial form $p(\mathbf{x})$ inside each triangle of the three-directional mesh. This polynomial, which is obtained by the sums of (15), can be precomputed, stored, and only evaluated at the end. The following code in C-language for $\chi^{2}$ may serve as a template: coordinates are first folded in the sector $[0, \pi / 2]$, then in $[0, \pi / 3]$, and finally in $[0, \pi / 6]$. This is done conveniently with the coordinates $(u, v)$. The coordinates $(g=u-v / 2, v)$ in the orthogonal basis $\left(\mathbf{r}_{1},\left(\mathbf{r}_{2}+\mathbf{r}_{3}\right) / 2\right)$ are the most appropriate for having short polynomials with rational coefficients in each triangle. 


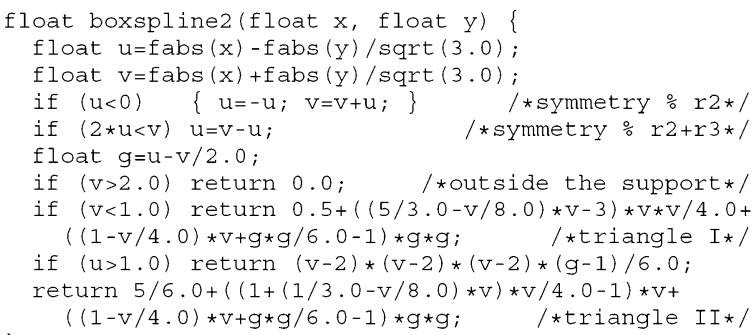

\section{CONCLUSION}

We proposed a new characterization of the three-directional box-splines, based on a Green function of the differential operator adapted to the hexagonal lattice. Together with a finite difference filter that acts as a localization operator on the generating function, this provides us with new explicit analytical formulas for the three-directional box-splines. This characterization also leads to particularly easy and efficient implementations. We provided the Matlab source code for the generic case and a further optimized C-code for the case $n=2$. The latter one could be particularly interesting for high-quality visualization of data sampled on a hexagonal lattice.

Finally, we note that these box-splines can be expressed on any lattice with matrix $\mathbf{R}^{\prime}$, and not only on the hexagonal one, by the simple change of basis $\chi^{n}\left(\mathbf{R R}^{\prime-1} \mathbf{X}\right)$.

\section{APPENDIX \\ PROOF OF THE PROPOSITION}

We verify whether $\rho^{n}$ of (9) is a Green function of $\mathrm{L}^{n}$, i.e., we need $\mathrm{L}^{n}\left\{\rho^{n}\right\}(\mathbf{x})=\delta(\mathbf{x})$. First, we introduce the vectors

$$
\mathbf{r}_{1}^{\perp}=\left[\begin{array}{c}
1 \\
\frac{1}{\sqrt{3}}
\end{array}\right], \quad \mathbf{r}_{2}^{\perp}=\left[\begin{array}{c}
1 \\
-\frac{1}{\sqrt{3}}
\end{array}\right], \quad \mathbf{r}_{3}^{\perp}=\left[\begin{array}{c}
0 \\
\frac{2}{\sqrt{3}}
\end{array}\right]
$$

which allow us to express the dual bases of $\left(\mathbf{r}_{2}, \mathbf{r}_{3}\right)$ and $\left(\mathbf{r}_{1}, \mathbf{r}_{3}\right)$ as $\left(\mathbf{r}_{3}^{\perp}, \mathbf{r}_{2}^{\perp}\right)$ and $\left(-\mathbf{r}_{3}^{\perp}, \mathbf{r}_{1}^{\perp}\right)$, respectively. For example, the coordinates of $\mathbf{x}$ in $\left(\mathbf{r}_{2}, \mathbf{r}_{3}\right)$ are $\left(\left\langle\mathbf{x}, \mathbf{r}_{3}^{\perp}\right\rangle,\left\langle\mathbf{x}, \mathbf{r}_{2}^{\perp}\right\rangle\right)$.

We now derive the Fourier expression of $\mu^{n_{1}, n_{2}}$, which we first rewrite as

$$
\begin{aligned}
\mu^{n_{1}, n_{2}}\left(x_{1}, x_{2}\right)= & \left(x_{2}\right)_{+}^{0} \mu^{n_{1}, n_{2}}\left(x_{1}, x_{2}\right) \\
& +\left(-x_{2}\right)_{+}^{0} \mu^{n_{1}, n_{2}}\left(x_{1}, x_{2}\right) \\
= & \left(\left\langle\mathbf{x}, \mathbf{r}_{3}^{\perp}\right\rangle\right)_{+}^{n_{1}}\left(\left\langle\mathbf{x}, \mathbf{r}_{2}^{\perp}\right\rangle\right)_{+}^{n_{2}} \\
& +\left(\left\langle\mathbf{x},-\mathbf{r}_{3}^{\perp}\right\rangle\right)_{+}^{n_{1}}\left(\left\langle\mathbf{x}, \mathbf{r}_{1}^{\perp}\right\rangle\right)_{+}^{n_{2}} .
\end{aligned}
$$

From distribution theory, we know the Fourier transform of the one-sided power function

$$
(x)_{+}^{n} \stackrel{\mathcal{F}}{\longleftrightarrow} \frac{n !}{(j \omega)^{n+1}}+\mathcal{D}(\boldsymbol{\omega})
$$

where $\mathcal{D}$ is essentially the $n$th derivative of Dirac. This term can be omitted since it does not have any influence when applying a differential operator of order $n$ (continuous or discrete) to $(x)_{+}^{n}$ (see also [2, App. C]).
Hence, using a tensor product and a change of basis from $\left(\mathbf{e}_{1}, \mathbf{e}_{2}\right)$ to $\left(\mathbf{r}_{2}, \mathbf{r}_{3}\right)$ (with Jacobian $\left.\left|\operatorname{det}\left[\mathbf{r}_{2} \mathbf{r}_{3}\right]\right|=\sqrt{3} / 2\right)$, we get

$$
\left(x_{2}\right)_{+}^{0} \mu^{n_{1}, n_{2}} \stackrel{\mathcal{F}}{\longleftrightarrow} \frac{\sqrt{3} / 2}{\left(j\left\langle\boldsymbol{\omega}, \mathbf{r}_{2}\right\rangle\right)^{n_{1}+1}\left(j\left\langle\boldsymbol{\omega}, \mathbf{r}_{3}\right\rangle\right)^{n_{2}+1}} .
$$

Similarly, the Fourier transform of $\left(-x_{2}\right)_{+}^{0} \mu^{n_{1}, n_{2}}$ is obtained by replacing $\mathbf{r}_{2}$ by $\mathbf{r}_{1}$ in (19).

We now define the functions $\gamma^{n_{1}, n_{2}, n_{3}}$, for any integers $n_{1}$, $n_{2}$, and $n_{3}$ as

$$
\gamma^{n_{1}, n_{2}, n_{3}} \stackrel{\mathcal{F}}{\longleftrightarrow} \frac{\frac{\sqrt{3}}{2}}{\left(j\left\langle\boldsymbol{\omega}, \mathbf{r}_{1}\right\rangle\right)^{n_{1}}\left(j\left\langle\boldsymbol{\omega}, \mathbf{r}_{2}\right\rangle\right)^{n_{2}}\left(j\left\langle\boldsymbol{\omega}, \mathbf{r}_{3}\right\rangle\right)^{n_{3}}}
$$

We recognize $\rho^{n}=\gamma^{n, n, n}, n \geq 1$. Using the property $\mathbf{r}_{3}=$ $\mathbf{r}_{1}+\mathbf{r}_{2}$, we can further obtain the following recurrence relation, for $n_{1} \geq 1, n_{2} \geq 1, n_{3} \geq 0$ :

$$
\gamma^{n_{1}, n_{2}, n_{3}}=\gamma^{n_{1}-1, n_{2}, n_{3}+1}+\gamma^{n_{1}, n_{2}-1, n_{3}+1} .
$$

By recurrence on $n_{1}+n_{2}$, we can also show that

$$
\begin{aligned}
\gamma^{n_{1}, n_{2}, n_{3}} & =\sum_{i=0}^{n_{1}-1}\left(\begin{array}{c}
n_{2}-1+i \\
i
\end{array}\right) \gamma^{n_{1}-i, 0, n_{2}+n_{3}+i} \\
& +\sum_{i=0}^{n_{2}-1}\left(\begin{array}{c}
n_{1}-1+i \\
i
\end{array}\right) \gamma^{0, n_{2}-i, n_{1}+n_{3}+i} .
\end{aligned}
$$

In the case of $\rho^{n}$, we have

$$
\rho^{n}=\sum_{i=0}^{n-1}\left(\begin{array}{c}
n-1+i \\
i
\end{array}\right)\left(\gamma^{n-i, 0,2 n+i}+\gamma^{0, n-i, 2 n+i}\right) .
$$

Finally, we identify the function $\mu^{n_{1}, n_{2}}$ as

$$
\mu^{n_{1}, n_{2}}=\gamma^{0, n_{1}+1, n_{2}+1}+\gamma^{n_{1}+1,0, n_{2}+1}
$$

which results in (9).

\section{REFERENCES}

[1] M. Unser and T. Blu, "Cardinal exponential splines: Part I-Theory and filtering algorithms," IEEE Trans. Signal Process., vol. 53, no. 4, pp. 1425-1438, Apr. 2005

[2] D. Van De Ville, T. Blu, M. Unser, W. Philips, I. Lemahieu, and R. Van De Walle, "Hex-spline: A novel family for hexagonal lattices," IEEE Trans. Image Process., vol. 13, no. 6, pp. 758-772, Jun. 2004.

[3] M. Unser, "Splines: A perfect fit for signal and image processing," IEEE Signal Process. Mag., vol. 16, no. 6, pp. 22-38, Nov. 1999.

[4] R. M. Mersereau, "The processing of hexagonally sampled two-dimensional signals," Proc. IEEE, vol. 67, no. 6, pp. 930-949, Jun. 1979.

[5] D. P. Petersen and D. Middleton, "Sampling and reconstruction of wavenumber-limited functions in N-dimensional Euclidean spaces," Inf. Control, vol. 5, pp. 279-323, 1962.

[6] C. de Boor, K. Höllig, and S. Riemenschneider, Box Splines. Berlin, Germany: Springer-Verlag, 1993, vol. 98, Applied Mathematical Sciences.

[7] H. Prautzsch and W. Boehm, "Box splines," in Handbook of Computer Aided Geometric Design. Berlin, Germany: Springer-Verlag, 2001.

[8] E. Cohen, T. Lyche, and R. Riesenfeld, "Discrete box splines and refinement algorithms," Comput. Aided Geom. Des., vol. 1, pp. 131-141, 1984.

[9] W. Dahmen and C. A. Michelli, "Subdivision algorithms for the generation of box-spline surfaces," Comput. Aided Geom. Des., vol. 1, pp. $115-129,1984$.

[10] M.-J. Lai, "Fortran subroutines for B-nets of box splines on three and four directional meshes," Numer. Algorithms, vol. 2, pp. 33-38, 1992.

[11] C. D. Boor, "On the evaluation of box splines," Numer. Alg., vol. 5, pp. 5-23, Mar. 1993.

[12] L. Kobbelt, "Stable evaluation of box-splines," Numer. Alg., vol. 14, no. 4, pp. 377-382, 1997. 